\title{
BMJ Open Efficacy of a standardised acupuncture approach for women with bothersome menopausal symptoms: a pragmatic randomised study in primary care (the ACOM study)
}

\author{
Kamma Sundgaard Lund, ${ }^{1}$ Volkert Siersma, ${ }^{2}$ John Brodersen, ${ }^{1,3}$ \\ Frans Boch Waldorff ${ }^{4}$
}

To cite: Lund KS, Siersma V, Brodersen J, et al. Efficacy of a standardised acupuncture approach for women with bothersome menopausal symptoms: a pragmatic randomised study in primary care (the ACOM study). BMJ Open 2019;9:e023637. doi:10.1136/ bmjopen-2018-023637

- Prepublication history and additional material for this paper are available online. To view these files, please visit the journal online (http://dx.do org/10.1136/bmjopen-2018023637).

Received 18 April 2018 Revised 17 December 2018 Accepted 21 December 2018

Check for updates

(c) Author(s) (or their employer(s)) 2019. Re-use permitted under CC BY-NC. No commercial re-use. See rights and permissions. Published by BMJ.

For numbered affiliations see end of article.

Correspondence to Dr Kamma Sundgaard Lund; kaml@sund.ku.dk

\section{ABSTRACT}

Objective To investigate the efficacy of a standardised brief acupuncture approach for women with moderate-tosevere menopausal symptoms.

Design Randomised and controlled, with 1:1 allocation to the intervention group or the control group. The assessor and the statistician were blinded.

Setting Nine Danish primary care practices.

Participants 70 women with moderate-to-severe menopausal symptoms and nine general practitioners with accredited education in acupuncture.

Intervention The acupuncture style was western medical with a standardised approach in the predefined acupuncture points CV-3, CV-4, LR-8, SP-6 and SP-9. The intervention group received one treatment for five consecutive weeks. The control group was offered treatment after 6 weeks.

Main outcome measures Outcomes were the differences between the randomisation groups in changes to mean scores using the scales in the MenoScores Questionnaire, measured from baseline to week 6 . The primary outcome was the hot flushes scale; the secondary outcomes were the other scales in the questionnaire. All analyses were based on intention-to-treat analysis.

Results 36 participants received the intervention, and 34 participants were in the control group. Four participants dropped out before week 6. The acupuncture intervention significantly decreased hot flushes: $\Delta-1.6(95 \% \mathrm{Cl}[-2.3$ to -0.8$]$; $\mathrm{p}<0.0001)$, day-and-night sweats: $\Delta-1.2$ (95\% Cl $[-2.0$ to -0.4$] ; p=0.0056)$, general sweating: $\Delta$ $-0.9(95 \% \mathrm{Cl}[-1.6$ to -0.2$] ; \mathrm{p}=0.0086)$, menopausalspecific sleeping problems: $\Delta-1.8(95 \% \mathrm{Cl}[-2.7$ to $-1.0]$; $p<0.0001)$, emotional symptoms: $\Delta-3.4(95 \%$ $\mathrm{Cl}[-5.3$ to -1.4$] ; \mathrm{p}=0.0008)$, physical symptoms: $\Delta$ $-1.7(95 \% \mathrm{Cl}[-3$ to -0.4$] ; p=0.010)$ and skin and hair symptoms: $\Delta-1.5$ (95\% Cl [ -2.5 to -0.6$] ; p=0.0021)$ compared with the control group at the 6-week followup. The pattern of decrease in hot flushes, emotional symptoms, skin and hair symptoms was already apparent 3 weeks into the study. Mild potential adverse effects were reported by four participants, but no severe adverse effects were reported.

Conclusions The standardised and brief acupuncture treatment produced a fast and clinically relevant reduction

\section{Strengths and limitations of this study}

- This study has high methodological quality, allocation concealment, adequate power, a validated outcome measure, sufficient and transparent reporting leading to high external validity.

- The study had high participants adherence supporting that the intervention was manageable and well tolerated.

- Since the intervention was pragmatic, standardised and brief, the applicability of the findings is high and might have a good chance of being implemented, which could lead to new treatment options for menopausal women.

- At present, no sufficient acupuncture placebo comparator exists, which is a major limitation in acupuncture studies, this study included.

in moderate-to-severe menopausal symptoms during the six-week intervention. No severe adverse effects were reported.

Trial registration number NCT02746497; Results.

\section{INTRODUCTION}

Experience of menopausal symptoms is very common and has been shown to affect quality of life, health status, work productivity and use of health services. ${ }^{1-3}$ The majority of women experience menopause in their early $50 \mathrm{~s}^{4}$ and have menopausal symptoms for 4-5years on average. ${ }^{4-7}$ The most prominent symptom of menopause is hot flushes, which affect around $75 \%$ of menopausal women ${ }^{568}$ and is reported as very distressing by $10 \%-20 \% .^{5}$ Other reported menopausal symptoms are night sweats, emotional vulnerability, sleep disturbances, fatigue, cognitive changes, joint pain, vaginal dryness and loss of sexual desire. ${ }^{459}$ 
Hormone therapy (HT) relieves menopausal symptoms,${ }^{1011}$ but long-term HT is associated with an increased risk of breast cancer and thromboembolic disorders. ${ }^{11-14}$ Hence, many menopausal women avoid HT. Non-hormonal-based treatments such as clonidine, gabapentin and antidepressants may also reduce menopausal symptoms. However, these drugs have frequent adverse effects such as sleep disturbance, dizziness, nausea, fatigue, dry mouth and constipation. ${ }^{4581516}$ Non-pharmaceutical treatments, for example, relaxation, exercise, herbal remedies and diets containing phytoestrogens have been suggested, although there is a lack of knowledge about dose, duration and for herbal remedies and phytoestrogens, drug interactions and adverse effects. There is currently no convincing evidence of any beneficial effect from these treatments. ${ }^{4815-17}$

Several studies have demonstrated the effects of acupuncture on menopausal symptoms, ${ }^{15-20}$ but they have been criticised for methodological limitations, for example, poor design, inadequate sample size, inadequate control or placebo groups, absence of standardised protocols and a lack of data on adverse effects. ${ }^{18}{ }^{19}$ Furthermore, due to different methods and a lack of validation of some outcome measures, comparison of results is difficult. ${ }^{18} 19$ Therefore, further high-quality randomised acupuncture trials are needed. ${ }^{18} 19$ Although the use of acupuncture differs between countries, it is sought by many patients ${ }^{21-24}$ and practised by a substantial number of physicians, especially general practitioners (GPs)..$^{225-27}$ If a clinically relevant effect on menopausal symptoms from acupuncture is demonstrated, this treatment may be considered for implementation in primary healthcare, leading to new options for menopausal women who cannot or do not wish to use HT.

We hypothesised that a brief and standardised acupuncture treatment could reduce moderate-to-severe menopausal symptoms, and in particular, it could have a clinically relevant effect in the reduction of hot flushes. Therefore, the objective of this study was to investigate the efficacy of a standardised brief acupuncture approach for women with moderate-to-severe menopausal symptoms, primarily the efficacy on hot flushes measured as change from baseline to week 6 .

\section{METHOD}

\section{Trial design}

The study was a randomised controlled trial (RCT) with a 1:1 allocation to the intervention or the control group. A detailed description of the methods used in the present study are found in the published protocol. ${ }^{28}$

\section{Settings and acupuncturists}

The study took place in nine primary care practices in both urban and rural settings. The acupuncturists were nine GPs, and all but one were educated in acupuncture by the Danish Society for Evidence-based Acupuncture (DSEA) or the Danish Medical Acupuncture Society
(DMAS). One GP had acupuncture training in Sri Lanka before DSEA and DMAS were formed. Participating GPs had, on average, 153 hours of acupuncture education (range 80-300) and had practised acupuncture for 14 years (range $4-38$ years).

The first author (KSL) held an individual meeting with each of the GP acupuncturists and provided them with the study protocol, an overview of the predefined acupuncture points and a written manual with precise instructions for treatment. KSL asked the GP acupuncturists to behave neutrally and to provide only the specified acupuncture treatment and no other treatment or counselling. A 2.5-hour refresher course on the predefined acupuncture points and techniques was offered, and four GPs attended this course.

\section{Participants}

Women were recruited through local newspapers, general practices close to the participating GP acupuncturist, the DSEA and the DMAS. Recruitment took place between late September 2016 and mid-December 2016.

Inclusion criteria: women aged 40-65 years with moderate-to-severe hot flushes (score $\geq 4$ on a validated scale measuring hot flushes (MenoScores Questionnaire $[\mathrm{MSQ}]^{29}$ and online supplementary appendix 1 ), intact cognitive function and a valid email address. Before enrolment, participants gave written informed consent. Exclusion criteria: women who had had a hysterectomy and/or bilateral oophorectomy; women whose alcohol consumption exceeded 21 drinks per week; who used prescribed sleeping pills and/or prescribed sedatives; who had previously been diagnosed with breast, endometrial, cervical or ovarian cancer; who had been diagnosed with other severe cancer disease within the past 5 years; who had heart valve disease; who were insulin dependent and/or had poorly controlled diabetes mellitus; who were diagnosed with thyroid disease; who were under investigation for serious disease, for example, cancer; who had received acupuncture treatment within the past 6 months; who had been pregnant or had been breast feeding within the past 2 years; who were participating in another trial or had participated in another trial in the 2 weeks before screening for eligibility; who, within the past 4 weeks, had used one or more of the following treatments: systemic HT, hormonal intrauterine device, antidepressants and/ or antiepileptics; and who had received other medical treatment for hot flushes (eg, clonidine), herbal remedies/alternative treatments for menopausal symptom or corticosteroids (the use of inhaled steroids was not an exclusion criterion).

Enrolled participants were provided with oral and written information about the study. ${ }^{30}$ Participation was voluntary, and there was no payment for taking part. Participants could withdraw their consent at any time. The first author carried out assessment of eligibility, obtained informed consent and collected baseline characteristic data. 


\section{Table 1 Acupuncture points and location \\ CV-3 Anterior midline, 1 cun* proximal to the symphysis. Insertion depth; perpendicularly $0.5-1 \mathrm{~cm}$. \\ CV-4 Anterior midline, 1 cun proximal to CV-3 and 3 cun inferior to the umbilicus. Insertion depth; perpendicularly $0.5-1 \mathrm{~cm}$. \\ LR-8 Medial side of the knee, in the depression anterior/ medial to the tendons of semimembranosus and the semitendinosus muscles, at the medial end of the popliteal crease. Insertion depth; perpendicularly $1.5-2 \mathrm{~cm}$.

\begin{tabular}{|c|c|}
\hline SP-9 & $\begin{array}{l}\text { Under the medial condyle of tibia in a depression } \\
\text { between the posterior tibia and } \mathrm{m} \text {. gastrocnemius. } \\
\text { Insertion depth; perpendicularly } 2-3 \mathrm{~cm} \text {. }\end{array}$ \\
\hline SP-6 & $\begin{array}{l}\text { Three cun proximal to the prominent part of the } \\
\text { medial malleoli, on the medial and posterior border } \\
\text { of the tibia. Insertion depth; perpendicularly } 1-3 \mathrm{~cm} \text {. }\end{array}$ \\
\hline
\end{tabular}

${ }^{*} \mathrm{~A}$ cun is an acupuncture measurement unit. One cun corresponds to the width of the study subject's thumb.

\section{Intervention}

All participants were offered one treatment per week for 5 weeks by a GP acupuncturist in their local area. The intervention group received their treatment in the first 5 weeks after enrolment in the study. At present, no validated acupuncture placebo comparator exists, ${ }^{31} 32$ and we decided to use a control group instead. The control group received their treatment after 6 weeks. Hence, this RCT is evaluated over a 6-week study period (online supplementary appendix 2; time schedule). No other treatment (medicine or alternative remedies) for menopausal symptoms was allowed in any of the groups beginning 4 weeks prior to enrolment until study week 11. The acupuncture style we used was western medical acupuncture (WMA), ${ }^{32} 33$ with a standardised approach and predefined acupuncture points (table 1 and online supplementary appendix 3), based on reports from experienced western medical acupuncturists. ${ }^{34}$

A complete acupuncture session should not last more than $15 \mathrm{~min}$, including insertion of needles, retention time, removal of needles and documentation. Disposable sterile (Plandent) needles, size $0.30 \times 30 \mathrm{~mm}$, were inserted perpendicularly and rotated manually for a few seconds to elicit 'de-qi' (needle sensation, a feeling of heaviness around the acupuncture point).$^{33}$ The predefined points were CV-3, CV-4, LR-8, SP-6 and SP-9 (table 1). A total of eight points was used, as LR-8, SP-6 and SP-9 were given bilaterally. Needle retention time was $10 \mathrm{~min}$.

After each treatment, the GP acupuncturist completed a documentation scheme with the date, documentation for insertion of each of the needles and whether 'de-qi' was obtained. After the final treatment, the completed documentation was sent to the first author.

\section{Outcome}

The study's outcomes were the differences between the randomisation groups in the mean change over the 6-week study period measured in the scales of the MSQ. ${ }^{29}$ The MSQ is a content-specific patient-reported outcome measure (PROM) with high-content validity and adequate psychometric properties measuring bothersome menopausal symptoms. The MSQ encompasses 11 scales and one single item (51 items in total), measuring different menopausal domains of bothersome symptoms (online supplementary appendix 1). The MSQ scales are constructed such that higher scores denote more bothersome symptoms.

The primary outcome was the hot flushes (HF) scale, secondary outcomes were the remaining MSQ scales: day-and-night sweats (DNS), general sweating (GS), menopausal-specific sleeping problems (MSSP), emotional symptoms (EM), memory changes (MEM), skin and hair symptoms (SH), physical symptoms (PHY), abdominal symptoms (ABD), urinary and vaginal symptoms (URIN), sexual symptoms (SEX) and the singleitem tiredness (TR).

Of these MSQ scales, the HF, DNS, GS and MSSP scales are most related to menopause, while the other MSQ scales are more related to general ageing or other life events. $^{29}$

\section{Assessments and follow-up}

All participants received the MSQ by email in study weeks $0,3,6,8,11$ and 26 (online supplementary appendix 2). Week 0 (before randomisation), week 3 (intermediate assessment) and week 6 (final assessment) are reported in the present manuscript. In the period when participants were receiving acupuncture treatment, we asked them to complete the MSQ 1-2 days before the third treatment and 1 week after the fifth and last treatment (online supplementary appendix 2). Participants completed and returned the MSQ electronically. Reminders were sent within 1-2 days, if the participant did not return a completed MSQ within the scheduled time. Additionally, participants in the intervention group were asked about adverse effects at the 3-week and 6-week follow-up. After the final treatment, we asked the intervention group if, in general, they had experienced a beneficial effect from the acupuncture treatment.

\section{Sample size}

The necessary sample size for the RCT was determined from reports on the primary outcome $\mathrm{HF}$ and the two secondary outcome DNS and MSSP in the MSQ validation study. ${ }^{29}$ We considered a reduction in a scale score as clinically relevant if it corresponded to a reduction from 'a lot' to 'quite a bit' on a global item regarding whether the respondent was bothered by menopausal symptoms. In the MSQ validation study, women who were bothered 'a lot' had a mean score of 4.98 on the HF scale, and women who were bothered 'quite a bit' had a mean score of 3.48 on the HF scale. Both groups have an SD around 1.4. To detect such a reduction on the HF scale with $90 \%$ power, $5 \%$ level of significance and accounting for $20 \%$ dropouts, we needed to include 48 participants (24 
Table 2 Baseline characteristics for each group of women

\begin{tabular}{lll}
\hline & $\begin{array}{l}\text { Control } \\
(\mathbf{n}=\mathbf{3 4})\end{array}$ & $\begin{array}{l}\text { Intervention } \\
(\mathbf{n}=\mathbf{3 6})\end{array}$ \\
\hline $\begin{array}{l}\text { Age (years), mean (SD) } \\
\text { Age (years), } n(\%)\end{array}$ & $54.1(5)$ & $55.3(4)$ \\
$40-55$ & $13(38)$ & $17(47)$ \\
\hline $56-65$ & $21(62)$ & $19(53)$ \\
$\begin{array}{l}\text { Employment, } n(\%) \\
\quad \text { Employed }\end{array}$ & $31(91)$ & $32(89)$ \\
$\quad$ Unemployed & $3(9)$ & $4(11)$
\end{tabular}

Education, $n$ (\%)

\begin{tabular}{|c|c|c|}
\hline Vocational & $8(24)$ & $8(22)$ \\
\hline Short (<3 years) & $4(12)$ & $3(8)$ \\
\hline Long ( $\geq 3$ years) & $16(47)$ & $22(61)$ \\
\hline Other & $6(18)$ & $3(8)$ \\
\hline \multicolumn{3}{|l|}{ Household, $n$ (\%) } \\
\hline Living alone & $1(3)$ & $5(14)$ \\
\hline Living with others & $33(97)$ & $31(86)$ \\
\hline \multicolumn{3}{|l|}{ Physical activity, $n$ (\%) } \\
\hline No physical activity & $7(21)$ & $3(8)$ \\
\hline 1-3 times per week & $20(59)$ & $23(64)$ \\
\hline$\geq 4$ times per week & $7(21)$ & $10(28)$ \\
\hline \multicolumn{3}{|l|}{ Smoking, $n$ (\%) } \\
\hline Yes & $1(3)$ & $2(6)$ \\
\hline No & $33(97)$ & 34 (94) \\
\hline \multicolumn{3}{|l|}{ Alcohol, $n$ (\%) } \\
\hline No alcohol & 1 (3) & $8(22)$ \\
\hline$\leq 14$ units per week & $29(85)$ & $19(53)$ \\
\hline$>14$ units per week & $4(12)$ & $9(25)$ \\
\hline $\begin{array}{l}\text { Body mass index }\left(\mathrm{kg} / \mathrm{m}^{2}\right) \text {, } \\
\text { mean }(S D)\end{array}$ & $25.5(5)$ & $24.9(3)$ \\
\hline
\end{tabular}

Menstruation in the last year, $n(\%)$

$\begin{array}{lrr}\text { Yes } & 7(21) & 11(31) \\ \text { No } & 27(79) & 25(69) \\ \text { Number of births, } n(\%) & & \\ \quad \text { None } & 3(9) & 2(6) \\ \text { One } & 5(15) & 8(22) \\ \text { Two } & 20(59) & 18(50) \\ \quad \text { More than two } & 6(18) & 8(22) \\ \text { Incontinentia, } n(\%) & & \\ \quad \text { No } & 13(38) & 10(28) \\ \text { Yes } & 21(62) & 26(72) \\ \text { Chronic disease, } n(\%) & & \\ \quad \text { Yes } & 5(15) & 8(22) \\ \text { No } & 29(85) & 28(78)\end{array}$

Previous experience with alternative treatment, $n(\%)$

\begin{tabular}{rrr} 
No & $8(24)$ & $10(28)$ \\
\hline & Continued
\end{tabular}

Table 2 Continued

\begin{tabular}{ccc}
\hline & $\begin{array}{l}\text { Control } \\
(\mathbf{n}=\mathbf{3 4 )}\end{array}$ & $\begin{array}{l}\text { Intervention } \\
(\mathbf{n}=\mathbf{3 6 )}\end{array}$ \\
\hline \multicolumn{1}{|c|}{ Yes } & $26(77)$ & $26(72)$ \\
$\begin{array}{l}\text { Duration of hot flushes (years), } \\
\text { mean (SD) }\end{array}$ & $3.41(3)$ & $4.59(4)$ \\
\hline
\end{tabular}

Hot flushes (HF), $n$ (\%)

\begin{tabular}{lll}
$\mathrm{HF}=4$ & $20(59)$ & $15(42)$ \\
$\mathrm{HF}>4$ & $14(41)$ & $21(58)$ \\
\hline
\end{tabular}

participants in each group). To achieve a similar power on the DNS and MSSP scales, we needed 56 and 68 participants, respectively.

\section{Randomisation}

The allocation sequence was computer generated using SAS software (V.9.4) and kept by a person independent of the project organisation (affiliated to the Centre for Health Economic Research, University of Southern Denmark). After enrolment, the independent person allocated the participants to one of the two randomisation groups. The participants were subsequently referred to the acupuncturist. This process ensured that allocation could not be guessed or later changed, thereby securing allocation concealment. Randomisation was done in blocks, with random block sizes, and stratified by age (aged 40-55 years or 56-65 years) and level of symptoms (experiencing hot flushes 'quite a bit' or 'a lot').

\section{Statistical methods}

For each of the primary and secondary outcomes, the up to three assessments for each woman were modelled with a linear mixed model with a level for each time point for each randomisation group; the inherent correlation between observations on the same woman was accounted for by the inclusion of a subject-random effect. The effect of the intervention was estimated at week 3 and week 6 by the mean difference of the outcome beyond the difference already present at baseline and assessed by the appropriate Wald test in the model. The model additionally included as covariates the dichotomisations used in the stratification of the randomisation: age and level of symptoms. The analysis was done intention to treat. Four or more treatments were considered adequate adherence.

All outcomes were Rasch validated, which implies sufficiency; that is, the sum-score carries all information of the measurement. Therefore, a score on one of scales of 4 is more than a score of 3 and so on. This justifies the use of the scores of the scales as continuously valued outcome variables in our analyses. A check of the assumptions of the linear regression analyses, aided by the Central Limit Theorem, justifies the comparison of the untransformed mean difference in scores and the use of the asymptotic Wald t-tests. Finally, the mixed model approach constitutes a first line defence against differential dropout. 


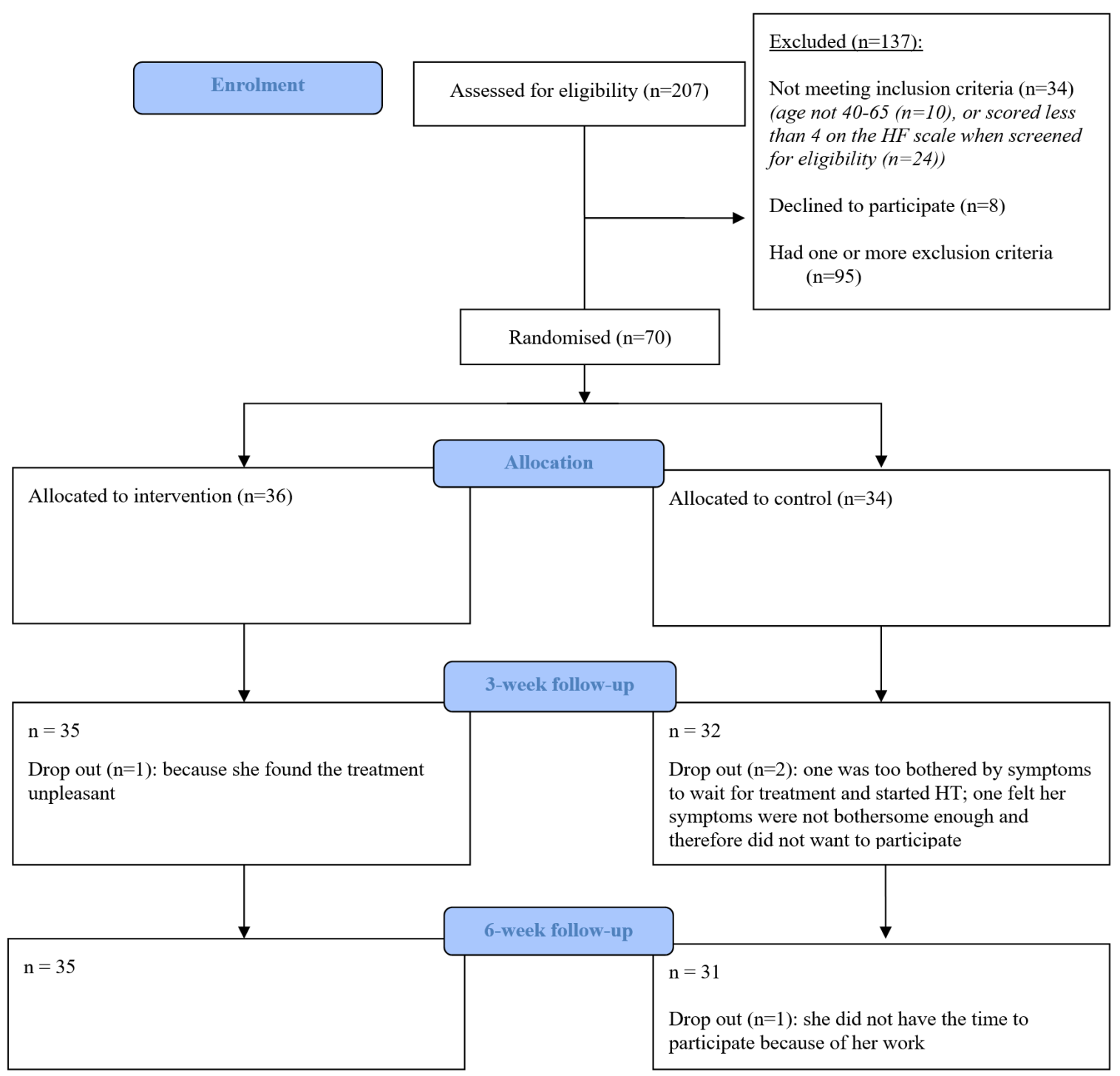

Figure 1 Trial flow. HF, hot flushes; HT, hormone therapy.

The statistical significance was assessed controlling for the false discovery rate at $5 \%$ with the method of Benjamini and Hochberg. ${ }^{35}$ SAS V.9.4 was used for the analyses.

\section{Blinding}

Statistician and outcome assessors were blinded until all analyses were completed. Participants and acupuncturist were not blinded.

\section{Patient involvement}

In the development of the research question, and in the design of the study, the development and content validity of the outcome measure (MSQ) was ensured by qualitative interviews with women who experienced bothersome menopausal symptoms. ${ }^{29}$ During this process, the relevance of this present study was also confirmed. Patients were not involved in the recruitment or conduction of the study. The burden of intervention was not assessed by the participants. When results are published, they will be disseminated to the Danish College of General
Practitioners, the DSEA, the DMAS, the project research homepage ${ }^{30}$ local newspaper and Danish women's lifestyle magazines.

\section{RESULTS}

We interviewed 207 women for eligibility, of which 70 met the inclusion criteria and were enrolled over a 3-month period: we allocated 36 participants to the intervention group and 34 participants to the control group. The number of participants treated by a single acupuncturist ranged from minimum 1 to maximum 16 (including the delayed treatment of the control group). No markedly differences in baseline characteristics between the randomisation groups were identified (table 2). Inspection of the residuals of the models did not reveal serious variance heterogeneity. Inspection of Cook's D did not reveal subjects that were particularly influential to the results. Four participants dropped out: one in the intervention 

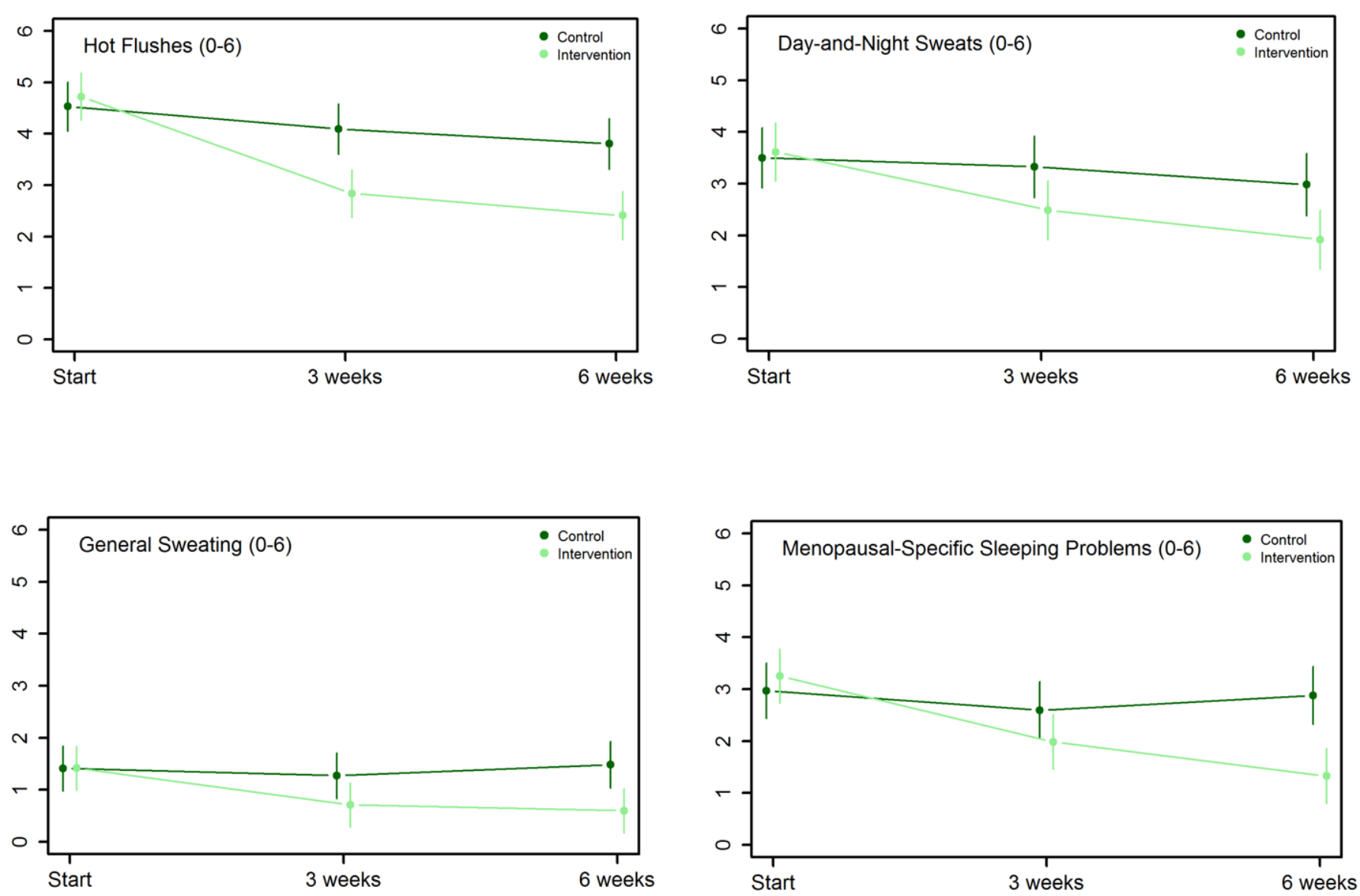

Figure 2 Development of the HF, DNS, GS and MSSP scales over the study period. DNS, day-and-night sweats; GS, general sweating; HF, hot flushes; MSSP, menopausal-specific sleeping problems. The error bars denote the $95 \% \mathrm{Cl}$ of the estimate of the outcome means for each randomisation group for each time point.

group and three in the control group (figure 1). The MSQ response rate was $100 \%$ for all remaining participants at all assessments points. The adherence to treatment was very high: 34 out of 36 received all five planned acupuncture treatments, and one received four out of five treatments. We collected primary data between October 2016 and February 2017.

The analyses of the observed raw scores demonstrated generally lower means in the intervention group compared with the control group when followed up (online supplementary appendix 4).

The developments in the MSQ scales across the two randomisation groups over the study period are presented in figures 2 and 3.

\section{Primary outcome}

The intervention group was significantly less bothered by HFs at 6 weeks: $\Delta-1.6$ (95\% CI [ -2.3 to -0.8$]$; $\mathrm{p}<0.0001)$. This difference was also statistically significant at 3 weeks: $\Delta-1.5$ (95\% CI [ -2.2 to -0.7$] ; \mathrm{p}=0.0002)$. (table 3 ).

\section{Secondary outcomes}

Statistically significant differences were identified at 6 weeks in the following secondary outcomes: DNS: $\Delta$ -1.2 (95\% CI [ -2.0 to -0.4$]$; $\mathrm{p}=0.0056)$; GS: $\Delta-0.9$ (95\%
CI $[-1.6$ to -0.2$] \mathrm{p}=0.0086)$; MSSP: $\Delta-1.8(95 \%$ CI $[-2.7$ to -1.0$] ; \mathrm{p}<0.0001)$; EM: $\Delta-3.4(95 \%$ CI $[-5.3$ to -1.4$]$; $\mathrm{p}=0.0008)$; PHY: $\Delta-1.7$ (95\% CI $[-3$ to -0.4$] ; \mathrm{p}=0.010)$ and SH $\Delta-1.5$ (95\% CI [-2.5 to -0.6$] ; \mathrm{p}=0.0021)$ (table 3$)$. This difference was also statistically significant at 3 weeks in EM: $\Delta-3.2(95 \%$ CI $[-5.1$ to -1.2$] ; \mathrm{p}=0.0015)$ and $\mathrm{SH}$ : $\Delta-1.4(95 \%$ CI $[-2.4$ to -0.5$] ; \mathrm{p}=0.0036)$ (table 3$)$.

In the intervention group, $80 \%$ of participants reported a general beneficial treatment effect after 6 weeks.

\section{Harms and adverse events}

No serious harms or adverse events were reported in the intervention group. Four participants reported mild potential adverse effects: one experienced tiredness and headache after treatment; another experienced more hot flushes in some of the weeks but reported this to be associated with increased stress in her personal and professional life; one had to urinate more frequently; and one experienced tingling in the leg where the needle had been placed.

Besides the four participants who reported mild potential adverse effects, one participant dropped out because she found the needling unpleasant (figure 1). However, this was not unexpected as acupuncture needling in some 

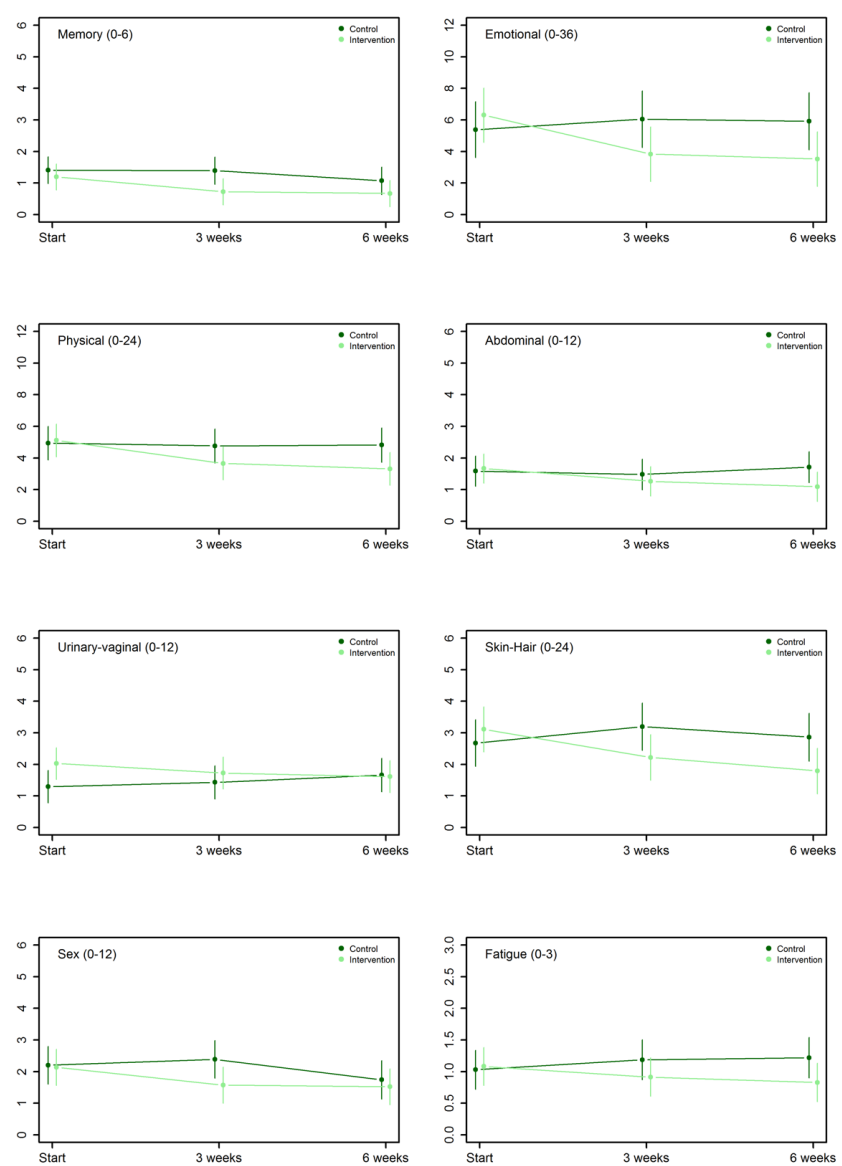

Figure 3 Development of the remaining (EM, MEM, SH, PHY, ABD, URIN and SEX) MSQ scales and the single item over the study period. ABD, abdominal symptoms; EM, emotional symptoms; MEM, memory changes; MSQ, MenoScores Questionnaire; PHY, physical symptoms; SEX, sexual symptoms; $\mathrm{SH}$, skin and hair symptoms; URIN, urinary and vaginal symptoms.

cases is experienced as a bit unpleasant (needles are inserted into the body which might be a bit unpleasant).

\section{DISCUSSION}

\section{Principal findings}

The standardised acupuncture treatment used in the present study reduced the HF, DNS, GS, MSSP, EM, PHY and $\mathrm{SH}$ scales after 5 weekly treatments, and the HF, EM and $\mathrm{SH}$ scales were reduced after two acupuncture treatments. The intervention did not significantly reduce the remaining MSQ scales, but we did also not expect to see this because this study was underpowered regarding these scales. Additionally, since significant reductions were only found in some of the scales, our findings emphasise that the intervention was targeted to menopausal symptoms and are not an artefact of general care. The acupuncture treatment was well tolerated: one participant dropped out, and only four participants reported mild potential adverse effects. No serious harms were reported.

\section{Strengths and weaknesses of the study}

The intervention period was relatively brief. However, it served to test a pragmatic, standardised and brief acupuncture approach manageable by both GPs and participants. Therefore, the suitability of the treatment outlined in this study in day-to-day primary care is high. The study had very high participant adherence: only 4 out of 70 participants dropped out. The remaining participants fulfilled the criteria for adequate treatment adherence and had a 100\% MSQ response rate, demonstrating that the intervention was well tolerated. The fact that the control group was also offered treatment after 6 weeks probably contributed to the high adherence rate. Finally, all participants were offered the same intervention, and cointerventions for menopausal symptoms were not allowed in the study period, thereby reducing the risk of performance bias. Not all menopausal women need or request treatment, and we believe this acupuncture intervention is most relevant to women who experience moderate-to-severe menopausal symptoms. We wanted to avoid outcomes confounded by cofactors such as severe illness, other medications or cointerventions, and since the study took place in primary care, the participants should reflect healthy women attending their GP requesting treatment for menopausal symptoms. We believe the participants are representative of such women and that our standardised and pragmatic intervention could easily be transferred to most clinical settings.

Another strength in this study was the use of a condition-specific PROM (MSQ) with high-content validity and adequate psychometric properties ensuring high construct validity of the study measurements. Moreover, data from the MSQ validation study ${ }^{29}$ was used to generate the power calculation based on relevant clinical effects that ensured adequate sample size. We did not use a physiological measurement, for example, skin conductance or temperature (which would probably have been technically difficult) because we believe PROMs are the most appropriate method to obtain information on participants' own perception of their symptoms. ${ }^{36}$ The recall time frame in this study was 1 week, which reduced the risk of recall bias.

The placebo effect plays an important role in all interventional studies and is influenced by expectations and beliefs. All GPs were certified acupuncturists and although they were instructed to behave neutrally, their beliefs in acupuncture could have affected their interaction with the participants and possibly have intensified a placebo effect. However, correct acupuncture techniques requires extensive training, and using GPs without such training would have been wrong and misleading. In addition, all participants were volunteers with presumably expectations of a beneficial effect. This might have enhanced the placebo effect in the intervention group and could have caused a nocebo effect in the control group. However, we did not see such deterioration in the control group. In fact, the control group showed a trend of improvement, in particular in the HF scale, which may 
Table 3 Differences in means, primary and secondary outcomes

\begin{tabular}{|c|c|c|c|c|c|}
\hline & \multirow{2}{*}{$\begin{array}{l}\text { Week } 0 \text { (baseline)* } \\
\Delta(95 \% \mathrm{Cl})\end{array}$} & \multicolumn{2}{|l|}{ Week 3† } & \multicolumn{2}{|l|}{ Week 6† } \\
\hline & & $\Delta(95 \% \mathrm{Cl})$ & $P$ value & $\Delta(95 \% \mathrm{Cl})$ & $P$ value \\
\hline Hot flushes & $0.1(-0.6$ to 0.7$)$ & $-1.5(-2.2$ to -0.7$)$ & $0.0002 \ddagger$ & $-1.6(-2.3$ to -0.8$)$ & $<0.0001 \ddagger$ \\
\hline Day-and-night sweats & $0.0(-0.9$ to 0.8$)$ & $-1(-1.8$ to -0.1$)$ & 0.024 & $-1.2(-2.0$ to -0.4$)$ & $0.0056 \ddagger$ \\
\hline General sweating & $0.0(-0.6$ to 0.6$)$ & $-0.6(-1.3$ to 0.1$)$ & 0.091 & $-0.9(-1.6$ to -0.2$)$ & $0.0086 \ddagger$ \\
\hline $\begin{array}{l}\text { Menopausal-specific sleeping } \\
\text { problems }\end{array}$ & $0.2(-0.5$ to 1$)$ & $-0.9(-1.7 ;-0.1)$ & 0.033 & $-1.8(-2.7$ to -1.0$)$ & $<0.0001 \ddagger$ \\
\hline Emotional symptoms & $1.0(-1.5$ to 3.5$)$ & $-3.2(-5.1$ to -1.2$)$ & $0.0015 \ddagger$ & $-3.4(-5.3$ to -1.4$)$ & $0.0008 \ddagger$ \\
\hline Memory changes & $-0.2(-0.8$ to 0.4$)$ & $-0.5(-1.0$ to 0.1$)$ & 0.11 & $-0.2(-0.8$ to 0.1$)$ & 0.49 \\
\hline Physical symptoms & $0.1(-1.5$ to 1.6$)$ & $-1.3(-2.6 ;-0.0)$ & 0.049 & $-1.7(-3$ to -0.4$)$ & $0.010 \ddagger$ \\
\hline Urinary and vaginal symptoms & 0.8 (0.1 to 1.5$)$ & $-0.4(-1.1$ to 0.3$)$ & 0.21 & $-0.8(-1.5$ to -0.1$)$ & 0.025 \\
\hline Abdominal symptoms & $0.1(-0.6$ to 0.8$)$ & $-0.3(-1$ to 0.4$)$ & 0.38 & $-0.7(-1.4$ to 0.0$)$ & 0.042 \\
\hline Skin and hair symptoms & $0.3(-0.7$ to 1.4$)$ & $-1.4(-2.4$ to -0.5$)$ & $0.0036 \ddagger$ & $-1.5(-2.5$ to -0.6$)$ & $0.0021 \ddagger$ \\
\hline Sexual symptoms & $-0.2(-1$ to 0.7$)$ & $-0.7(-1.4$ to -0.1$)$ & 0.032 & $-0.3(-0.8$ to 0.5$)$ & 0.69 \\
\hline Tiredness & $0.1(-0.3$ to 0.5$)$ & $-0.3(-0.8$ to 0.1$)$ & 0.15 & $-0.5(-0.9$ to 0.0$)$ & 0.049 \\
\hline
\end{tabular}

Differences in mean scores between the randomization groups at each of the follow-up time points.

Negative values $\Delta=$ less symptoms in the intervention group.

*Difference in mean score of intervention relative to control, adjusted for stratification factors (age and level of symptoms ['quite a bit' or 'a lot' $H F]$ ).

†Difference in mean score of intervention relative to control beyond the difference already present at week 0 (baseline), adjusted for stratification factors (age and level of symptoms ['quite a bit' or 'a lot' HF]).

$\ddagger$ Significant at a 0.01 level to control for the false discovery rate at 0.05 .

be explained by a regression to the mean. The lack of a sufficient acupuncture placebo comparator is a major limitation in acupuncture studies, including this study. In WMA theories, sham (placebo) acupuncture is not perceived as inactive but rather another, although less effective, form of needling. ${ }^{31}{ }^{32}$ Furthermore, a meta-analysis concluded that non-specific effects associated with sham acupuncture are often moderately large and might be larger than other placebo interventions. ${ }^{37}$ If sham is not inactive, a study testing sham versus real acupuncture is not a placebo-controlled study but rather a study testing two different types of acupuncture. Therefore, we decided to investigate the impact of acupuncture versus no treatment. An important weakness of the present study is that the identified positive effects from acupuncture treatment could be caused by a placebo effect and not a specific physiological effect of needling. However, our aim was not to distinguish between specific and non-specific effects of needling but to investigate the impact of acupuncture versus no treatment. We found that the acupuncture treatment used in this study had an important clinical effect. If we ignore these findings, due to a lack of knowledge about possible specific effects of acupuncture, women with moderate-to-severe menopausal symptoms could miss out on a low cost and effective treatment with only minor potential adverse effects.

One final limitation was that blinding of acupuncturists and participants in this study design was not possible. However, we secured blinding of the statistician and outcome assessors until all analyses were completed.

\section{Comparison with other studies}

Some previous studies have demonstrated real acupuncture to be significantly superior to sham acupuncture. ${ }^{38-40}$ However, a Cochrane review from 2013 regarding acupuncture for menopausal HFs found no significant differences between real acupuncture compared with sham but a beneficial effect of acupuncture compared with no treatment and that acupuncture was inferior to HT. The evidence was in general of poor quality, and further high quality studies were recommended. ${ }^{18}$ Results from two recent studies, one comparing real acupuncture with sham ${ }^{41}$ and one comparing acupuncture with no treatment, ${ }^{42}$ confirm the findings reported in the Cochrane review.

The present study demonstrates that acupuncture is significantly superior to no treatment. Our study was based on WMA theories, while most previous studies were based on traditional Chinese medicine theories and diagnoses ${ }^{18} 2138-46$ involving the concepts of yin/ yang and circulation of qi. ${ }^{32}{ }^{33}$ Most previous studies had longer intervention periods and/or more treatment sessions $^{21}{ }^{39-50}$ and several studies used individualised treatment with variation in the selection of acupuncture points. $^{2140424346}$ Tailored treatments might be a truer reflection of the actual clinical context. However, in an RCT, we believe that treatment should be standardised so that the intervention can be replicated. Some studies differ from this study by including patients treated for breast cancer, ${ }^{38} 3944$ which makes it difficult to compare results. In contrary to our study, some studies 
allowed other cointerventions (eg, adjuvant antihormone therapy, cystostaticum, clonidine, antidepressants, HT or other alternative remedies), ${ }^{213839414244}$ which might have affected their outcomes. Finally, some studies assessed other relevant secondary outcomes such as quality of life, hot flush interference and sleep quality, and one study also assessed plasma oestradiol. Most studies used self-reported outcomes, but in several of them, the validation of the outcome measures was lacking, unclear or not reported. ${ }^{38394455}$ We did not use a quality of life measure, because we did not find a Rasch validated quality of life instrument for our target group. The MSQ validation ensured that all aspects considered important by menopausal women themselves were covered by the MSQ scales. We would expect that a reduction in the HF, DNS, GS, MSSP, EM, PHY and SH scales may ultimately have a positive indirect impact on a menopausal woman's overall sense of well-being and quality of life. To our knowledge, this study is the only one to use a PROM that is condition specific with high-content validity and psychometrically Rasch validated.

\section{Meaning of the study: possible explanations and implications for clinicians and policymakers}

The lack of a proper acupuncture placebo comparator has major implications for conducting and interpreting acupuncture studies. Therefore, we need to continue the discussion about what level of evidence should be accepted as sufficient for a treatment to be considered effective. This is particularly the case when we cannot accurately explain the underlying mechanism behind the treatment, nor determine how much of the effect is caused by placebo. In addition, it is important to note that in the present study, the intervention was targeted at menopausal symptoms and subsequently demonstrated a targeted effect, not an improvement across all symptoms. We also need to take the balance between benefits and harms into consideration. There is strong evidence that acupuncture for menopausal symptoms is without serious harms. Thus, requirements for evidence of the efficacy of acupuncture treatment might be less rigorous. On the contrary, acupuncture treatment in a private setting (ie, outside a publicly funded healthcare system) might involve considerable personal expense and opportunity costs.

We consider the intervention in this study to be low cost, both to the individual and to the health system, but this needs to be further investigated and included in a discussion of value-based healthcare..$^{51}$

\section{Future research}

The long-term effect, the characteristics of women who benefit from acupuncture treatment, cost-effectiveness, the underlying mechanism of needling and the impact of placebo need further investigation.

\section{CONCLUSION}

A standardised acupuncture treatment gives women suffering from moderate-to-severe menopausal symptoms a clinically relevant reduction in HFs, DNS, GS, MSSP, EM, PHY and SH. Acupuncture for menopausal symptoms is a realistic option for women who cannot or do not wish to use HT. Women seeking acupuncture treatment for menopausal symptoms should be informed of the current evidence, and its limitations, so they can integrate this with personal preferences and values in their decision making. This study has high methodological quality, adequate power, a validated outcome measure and sufficient reporting leading to high validity of the study and findings. Furthermore, this study use a pragmatic, standardised and brief intervention that leads to findings that may have a higher chance of being implemented and thereby are more likely to lead to new treatment options for menopausal women.

\section{Author affiliations}

${ }^{1}$ Section of General Practice, Department of Public Health, University of Copenhagen, Copenhagen, Denmark

${ }^{2}$ Research Unit for General Practice, Department of Public Health, University of Copenhagen, Copenhagen, Denmark

${ }^{3}$ Primary Health Care Research Unit, University of Copenhagen, Copenhagen, Denmark

${ }^{4}$ The Research Unit for General Practice, Department of Public Health, University of Southern Denmark, Odense, Denmark

Acknowledgements We would like to thank the doctors and women who took part in this study, Palle Rosted and Danish Society for Evidence-based Acupuncture for inspiration and counselling.

Contributors FBW conceived the idea. All authors all took part in the design and planning of the study. KSL conducted the study supported by FBW, VS and JB. KSL drafted the manuscripts. FBW, VS and JB revised the entire manuscript critically and approved the final version for publication. The statistics was carried out by VS. FBW is guarantor for the study.

Funding The Idella Foundation, the University of Copenhagen, the Research Foundation of General Practice including the Foundation of Multipractice Studies.

Competing interests None declared.

Patient consent for publication Not required.

Ethics approval Approvals from the Committees on Health Research Ethics (H-16016365), the Committee of Multipractice Studies in General Practice (MPU 08-2016) and the Danish Data Protection Agency (SUND-2016-24) were obtained before enrolment. The study was conducted in accordance with the Declaration of Helsinki and Good Clinical Practice (ICH GCP).

Provenance and peer review Not commissioned; externally peer reviewed.

Data sharing statement All authors had access to and take responsibility for the data and analyses. Relevant and only anonymised data (PROM scores) can be available for research on reasonable request (kaml@sund.ku.dk). Data will be saved for 5 years. The protocol was published in March 2017.

Author note Please refer to reference 28 for full trial protocol.

Open access This is an open access article distributed in accordance with the Creative Commons Attribution Non Commercial (CC BY-NC 4.0) license, which permits others to distribute, remix, adapt, build upon this work non-commercially, and license their derivative works on different terms, provided the original work is properly cited, appropriate credit is given, any changes made indicated, and the use is non-commercial. See: http://creativecommons.org/licenses/by-nc/4.0/.

\section{REFERENCES}

1. Whiteley J, Wagner JS, Bushmakin A, et al. Impact of the severity of vasomotor symptoms on health status, resource use, and productivity. Menopause 2013;20:1-24.

2. Williams RE, Levine KB, Kalilani L, et al. Menopause-specific questionnaire assessment in US population-based study shows 
negative impact on health-related quality of life. Maturitas 2009;62:153-9.

3. Geukes M, van Aalst MP, Robroek SJ, et al. The impact of menopause on work ability in women with severe menopausal symptoms. Maturitas 2016;90:3-8.

4. Nelson HD. Menopause. Lancet 2008;371:760-70.

5. Stearns V, Ullmer L, López JF, et al. Hot flushes. Lancet 2002;360:1851-61.

6. Avis NE, Crawford SL, Greendale G, et al. Duration of menopausal vasomotor symptoms over the menopause transition. JAMA Intern Med 2015;175:531-9.

7. Col NF, Guthrie JR, Politi M, et al. Duration of vasomotor symptoms in middle-aged women: a longitudinal study. Menopause 2009;16:453-7.

8. Pachman DR, Jones JM, Loprinzi CL. Management of menopause-associated vasomotor symptoms: Current treatment options, challenges and future directions. Int $J$ Womens Health 2010;2:123-35.

9. Prairie BA, Wisniewski SR, Luther J, et al. Symptoms of depressed mood, disturbed sleep, and sexual problems in midlife women: cross-sectional data from the Study of Women's Health Across the Nation. J Womens Health 2015;24:119-26.

10. Maclennan AH, Broadbent JL, Lester S, et al. Oral oestrogen and combined oestrogen/progestogen therapy versus placebo for hot flushes. Cochrane Database Syst Rev 2004;4:Cd002978.

11. Sarri G, Davies M, Lumsden MA. Guideline Development Group. Diagnosis and management of menopause: summary of NICE guidance. BMJ 2015;351:h5746.

12. Prentice RL, Manson JE, Langer RD, et al. Benefits and risks of postmenopausal hormone therapy when it is initiated soon after menopause. Am J Epidemiol 2009;170:12-23.

13. Rossouw JE, Anderson GL, Prentice RL, et al. Risks and benefits of estrogen plus progestin in healthy postmenopausal women: principal results From the Women's Health Initiative randomized controlled trial. JAMA 2002;288:321-33.

14. Beral V, Banks E, Reeves G, et al. Breast cancer and hormonereplacement therapy: the Million Women Study. The Lancet 2003;362:1330-1.

15. Mintziori G, Lambrinoudaki I, Goulis DG, et al. EMAS position statement: Non-hormonal management of menopausal vasomotor symptoms. Maturitas 2015;81:410-3

16. Woyka J. Consensus statement for non-hormonal-based treatments for menopausal symptoms. Post Reprod Health 2017;23:71-5.

17. Daley A, MacArthur C, Mutrie N, et al. Exercise for vasomotor menopausal symptoms. Cochrane Database Syst Rev 2007;4:Cd006108

18. Dodin S, Blanchet C, Marc I, et al. Acupuncture for menopausal hot flushes. Cochrane Database Syst Rev 2013;7:Cd007410.

19. Smith CA, Carmady B. Acupuncture to treat common reproductive health complaints: An overview of the evidence. Auton Neurosci 2010;157:52-6.

20. Chiu HY, Pan CH, Shyu YK, et al. Effects of acupuncture on menopause-related symptoms and quality of life in women in natural menopause: a meta-analysis of randomized controlled trials. Menopause 2015;22:234-44.

21. Borud EK, Alraek T, White A, et al. The Acupuncture on Hot Flushes Among Menopausal Women (ACUFLASH) study, a randomized controlled trial. Menopause 2009;16:484-93.

22. Cramer H, Chung VC, Lauche R, et al. Characteristics of acupuncture users among internal medicine patients in Germany. Complement Ther Med 2015;23:423-9.

23. Hanssen B, Grimsgaard S, Launsø L, et al. Use of complementary and alternative medicine in the Scandinavian countries. Scand J Prim Health Care 2005;23:57-62.

24. Xue CC, Zhang AL, Lin V, et al. Acupuncture, chiropractic and osteopathy use in Australia: a national population survey. BMC Public Health 2008;8:105.

25. Rosted P. [The effect of acupuncture on musculoskeletal disorders] Ugeskr Laeger 2005;167:3573-7.

26. Mao JJ, Kapur R. Acupuncture in primary care. Prim Care 2010;37:105-17.
27. Aanjesen T, Senstad AC, Lystad N, et al. [Acupuncture--a complementary treatment in general practice]. Tidsskr Nor Laegeforen 2002;122:921-3.

28. Lund KS, Brodersen J, Siersma V, et al. The efficacy of acupuncture on menopausal symptoms (ACOM study): protocol for a randomised study. Dan Med J 2017;64

29. Lund KS, Siersma VD, Christensen KB, et al. Measuring bothersome menopausal symptoms: development and validation of the MenoScores questionnaire. Health Qual Life Outcomes 2018;16:97.

30. Kobenhavns Universitet. Forskningsenheden for Almen Praksis. http://almenpraksis.ku.dk/forskning/menopause/ (Accessed Oct 2017).

31. Lundeberg T, Lund I, Näslund J, et al. The Emperors sham wrong assumption that sham needling is sham. Acupunct Med 2008;26:239-42.

32. White A. Editorial Board of Acupuncture in Medicine. Western medical acupuncture: a definition. Acupunct Med 2009;27:33-5.

33. White A, Cummings M, Filshie J. An introduction to western medical acupuncture. London: Churchill Livingstone, 2008.

34. Rosted P. Akupunktur: på naturvidenskabeligt grundlag. 1st edn. Århus: Klim, 2003.

35. Benjamini Y, Hochberg Y. Controlling the false discovery rate: a practical and powerful approach to multiple testing. Journal of the Royal Statistical Society: Series B 1995;57:289-300.

36. Cappelleri JC, Zou KH, Bushmakin AG, et al. Patient-reported outcomes: Measurement implementation and interpretation. Boca Raton, FL: CRC Press, 2013.

37. Linde K, Niemann K, Schneider A, et al. How large are the nonspecific effects of acupuncture? A meta-analysis of randomized controlled trials. BMC Med 2010;8:75.

38. Bokmand $\mathrm{S}$, Flyger $\mathrm{H}$. Acupuncture relieves menopausal discomfort in breast cancer patients: a prospective, double blinded, randomized study. Breast 2013;22:320-3.

39. Hervik J, Miåland O. Acupuncture for the treatment of hot flashes in breast cancer patients, a randomized, controlled trial. Breast Cancer Res Treat 2009;116:311-6.

40. Nir Y, Huang MI, Schnyer R, et al. Acupuncture for postmenopausa hot flashes. Maturitas 2007;56:383-95.

41. Ee C, Xue C, Chondros P, et al. Acupuncture for menopausal hot flashes: a randomized trial. Ann Intern Med 2016;164:146-54.

42. Avis NE, Coeytaux RR, Isom S, et al. Acupuncture in Menopause (AIM) study: a pragmatic, randomized controlled trial. Menopause 2016;23:626-37.

43. Avis NE, Legault C, Coeytaux RR, et al. A randomized, controlled pilot study of acupuncture treatment for menopausal hot flashes. Menopause 2008;15:1070-8.

44. Deng G, Vickers A, Yeung S, et al. Randomized, controlled trial of acupuncture for the treatment of hot flashes in breast cancer patients. J Clin Oncol 2007;25:5584-90.

45. Frisk J, Carlhäll S, Källström A-C, et al. Long-term follow-up of acupuncture and hormone therapy on hot flushes in women with breast cancer: a prospective, randomized, controlled multicenter trial. Climacteric 2008;11:166-74.

46. Venzke L, Calvert JF, Gilbertson B. A randomized trial of acupuncture for vasomotor symptoms in post-menopausal women. Complement Ther Med 2010;18:59-66.

47. Kim KH, Kang KW, Kim DI, et al. Effects of acupuncture on hot flashes in perimenopausal and postmenopausal women-a multicenter randomized clinical trial. Menopause 2010;17:269-80.

48. Vincent A, Barton DL, Mandrekar JN, et al. Acupuncture for hot flashes: a randomized, sham-controlled clinical study. Menopause 2007;14:45-52

49. Kim DI, Jeong JC, Kim KH, et al. Acupuncture for hot flushes in perimenopausal and postmenopausal women: a randomised, shamcontrolled trial. Acupuncture in Medicine 2011;29:249-56.

50. Painovich JM, Shufelt CL, Azziz R, et al. A pilot randomized, single-blind, placebo-controlled trial of traditional acupuncture for vasomotor symptoms and mechanistic pathways of menopause. Menopause 2012;19:54-61.

51. Ee C, French SD, Xue CC, et al. Acupuncture for menopausal hot flashes: clinical evidence update and its relevance to decision making. Menopause 2017;24:980-7. 